\title{
Evaluar la transferencia de la formación en liderazgo directivo: un caso chileno
}

\section{Evaluating the Transfer of Directive Leadership Training: A Chilean Case Study}

Rosita Romero Alonso (*) https://orcid.org/0000-0003-2800-5092

Blanca Montt Fabres (*) https://orcid.org/0000-0001-8287-6409

Benigno Pino Díaz (*) https://orcid.org/0000-0003-3724-7552

Irma Riquelme Plaza (*) https://orcid.org/0000-0002-5040-9555

María Elena Gracia Romero (*) https://orcid.org/0000-0002-3430-9669

(*) Universidad Tecnológica de Chile INACAP

(Recibido: 16 de octubre de 2018; Aceptado para su publicación: 7 de enero de 2019)

Cómo citar: Romero, R., Montt, B., Pino, B., Riquelme, I. y Gracia, M. E. (2020). Evaluar la transferencia de la formación en liderazgo directivo: un caso chileno. Revista Electrónica de Investigación Educativa, 22, e19, 1-13. https://doi.org/10.24320/redie.2020.22.e19.2683

\section{Resumen}

Es necesario contar con evaluaciones sistemáticas que informen sobre el verdadero impacto de la formación continua en las prácticas educativas. El objetivo de esta investigación fue evaluar la transferencia del aprendizaje de las funciones directivas al contexto de desempeño de los participantes de un plan de formación en liderazgo directivo escolar en Chile. Utilizando una metodología mixta se analizaron los resultados de una encuesta aplicada a una muestra de 47 participantes que permitió observar el nivel de aplicación de los aprendizajes: luego, el estudio de 12 casos profundizó en la comprensión de los resultados arrojados en la primera fase. La autoconfianza y motivación generada en los participantes, producto de la validez de contenido del curso, se identificaron como factores clave en el proceso de transferencia, mientras que la falta de condiciones institucionales y la dificultad para asumir cargos directivos fueron los principales obtaculizadores.

Palabras clave: Evaluación, educación permanente, formación de directores, liderazgo.

\section{Abstract}

Systematic evaluations are necessary to report on the true impact of continuing education on educational practices. The objective of this research was to assess the learning transfer of managerial functions among participants of a directive leadership training plan to the contexts in which they work in Chile. A mixed-methods methodology was employed to analyze the results of a survey administered to a sample of 47 participants, which showed the extent to which learning is being applied. A subsequent study of 12 cases provided a deeper understanding of the results of this first stage. Self-confidence and participant motivation - a product of the content validity of the course - were identified as key factors in the transfer process, while a lack of institutional conditions and difficulty in taking up managerial positions were the main obstacles. 


\section{Introducción}

La formación -siempre en la encrucijada de los resultados- se perfila como el medio de generar cambios en la profesionalidad docente, lo que supone un desafío con relación a la pertinencia de los métodos y contenidos que realmente se traduzcan en nuevas prácticas educativas. Por otro lado, se evidencia que la inversión en formación no tiene relación con necesidades percibidas por los profesores, y por lo mismo carecen de impacto real en las instituciones escolares. Para las instituciones formadoras es complejo leer estas necesidades, así como valorar pertinentemente el impacto de los procesos formativos que desarrollan.

En el ámbito de la pedagogía existe una mirada de insatisfacción docente frente a la formación permanente (Díaz et al., 2015). Los procesos formativos con enfoque tradicional han sido la forma histórica de abordar la docencia, pero salir de modelos de entrenamiento academicistas es una necesidad (Bozu e Imbernón, 2016). La baja valoración a los procesos formativos se condice con el bajo impacto que le atribuyen a su incidencia en el quehacer pedagógico, recurriendo muchas veces a la repetición de modelos adquiridos incluso en el sistema escolar (Vaillant y Marcelo, 2015). A la vez, se indica que la valoración de los procesos formativos en ejercicio de la profesión docente es baja debido a la variabilidad en la calidad de los mismos (Ávalos, 2014).

Reconocer que la práctica pedagógica de los profesores no es sólo la consecuencia de lo aprendido en la formación sino que responde a un complejo entramado de condiciones sociales, culturales y personales que se entrelazan en un contexto educativo determinado (Ávalos, 2016) ha significado la apertura en Chile a la estructuración de un sistema formal de inducción basado en mentorías (Marcelo y Vaillant, 2017), reconociendo el aporte de una estrategia formativa no academicista. Sin embargo, no se observa esa misma perspectiva en los procesos formativos que acompañan el desarrollo de la profesionalidad docente para los directivos.

Por otra parte, conocer el impacto de los procesos formativos es parte de las tendencias actuales, enfoque que se inició en el mundo de las empresas (Grohmann y Kauffeld, 2013; Grossman y Salas, 2011). Las organizaciones invierten muchos de recursos para mejorar los conocimientos y habilidades de los empleados, por lo que es importante que este esfuerzo se retribuya en una real aplicación de lo aprendido en las prácticas (Blume et al., 2010; Hutchins et al., 2010). Si bien se trata de experiencias concentradas en el ámbito empresarial, poco a poco ha ido permeando al ámbito educativo, como las experiencias desarrolladas por Feixas et al. (2014) y Ornelas et al. (2016) en la educación superior.

En este contexto, se propuso la evaluación del caso particular de un plan formativo en habilidades de liderazgo orientado a directores en ejercicio y docentes que aspiran a asumir cargos directivos, desarrollado en el marco del Plan de Formación de Directores de Excelencia impulsado por el Ministerio de Educación chileno. Para ello se plantearon dos objetivos: 1) constatar en qué medida los participantes aplican los conocimientos y competencias en su ámbito cotidiano de desempeño, y 2) identificar los elementos que facilitan y obstaculizan de transferencia de dichas competencias a su quehacer educativo.

\subsection{Elementos teóricos de la evaluación de la transferencia de la formación}

Desde los años ochenta se cuenta con estudios sobre la evaluación de la transferencia de la formación (Cano, 2015; Feixas et al., 2015), que puede ser entendida como las posibilidades de aplicación en el contexto de trabajo de una serie de conocimientos, habilidades y actitudes aprendidas en un proceso formativo (Baldwin y Ford, 1988). Específicamente en el ámbito de la formación docente, Feixas et al. (2014) la definen como "...la aplicación efectiva y continuada en el lugar de trabajo de un conjunto de habilidades, conocimientos y concepciones aprendidas en un contexto de desarrollo docente." (p. 57).

En una búsqueda sobre cómo evaluar los procesos formativos destacan los modelos desarrollados por Baldwin y Ford (1988), Holton (2005) y Kirkpatrick y Kirkpatrick (2006). Los dos primeros centrados en explicar factores que influyen en el proceso de transferencia, mientras que el último es más bien una 
mirada que clasifica las iniciativas de evaluación en cuatro niveles considerando la satisfacción como el primero y más básico, el aprendizaje en el segundo nivel, la transferencia al ejercicio profesional en el tercero y el impacto en el cuarto. Evidentemente existe más información y se aplican con mayor frecuencia evaluaciones a los programas formativos que abarcan los niveles 1 y 2 (Cano, 2016; Feixas et al., 2014). La falta de financiamiento y de programación de estas evaluaciones es un obstáculo para su ejecución más extendida en los niveles superiores (Berrocal y Campos, 2014; Cano, 2015).

La completitud del modelo de Kirkpatrick y Kirkpatrick (2006) es reconocida por autores como Cano (2015) y Grohmann y Kauffeld (2013), quienes destacan la posibilidad de atender a distintas dimensiones con sus consecuencias en el corto y mediano plazo -aun cuando la dificultad para aplicar todos los niveles, particularmente el referido al impacto en la organización lo dificulta, tiene gran valor la posibilidad de medir la aplicación práctica de lo aprendido (Cano, 2015). Tampoco hay una necesaria equivalencia entre niveles, como lo ha demostrado el hecho de que un alto nivel de satisfacción o aprendizaje no necesariamente se traduzca en altos niveles de aplicación (Cano, 2015; Wijk et al., 2008).

\subsection{Factores que inciden en la transferencia de la formación}

La transferencia de la formación se ha estudiado detenidamente (Blume et al., 2010; Cano, 2016; Feixas et al., 2015; Grossman y Salas, 2011; Massenberg et al., 2017) identificando tres tipos de factores que influyen en que los aprendizajes sean finalmente aplicados en el contexto: los insumos de la formación (personas y características del proceso formativo), resultados del proceso formativo como tal y condiciones de transferencia del entorno de desempeño.

El costo y las posibilidades prácticas hacen difícil que las organizaciones puedan medir la diversidad de factores, por lo que los estudios se acotan a un grupo de variables que demuestran una relación fuerte, de acuerdo con Grossman y Salas (2011), serían las siguientes: la capacidad cognitiva de los alumnos (Blume et al., 2010), la percepción de autoeficacia (Burke y Hutchins, 2007; Veldada et al., 2007); la motivación (Chiaburu y Lindsay, 2008; Grohmann et al., 2014; Massenberg et al., 2017); la utilidad percibida respecto a los aportes de la formación o validez de contenido (Grohmann et al., 2014; Grohmann y Kauffeld, 2013; Veldada et al., 2007); el diseño de la formación para la transferencia o la formación en un entorno real (Burke y Hutchins, 2007; Grohmann et al., 2014; Grohmann y Kauffeld, 2013); el entorno o clima de transferencia (Blume et al., 2010; De Rijdt et al., 2013); el apoyo de supervisores en su entorno (Blume et al., 2010; Salas et al., 2006); el apoyo de sus compañeros (Blume et al., 2010; Salas et al., 2006); las posibilidades de transferencia que da el entorno (Burke y Hutchins, 2007; Salas et al., 2006) y el mantenimiento o refuerzo de la formación en el tiempo (Salas et al., 2006; Veldada et al., 2007).

De los factores identificados se ha mostrado una fuerte relación entre la motivación para transferir y todos los demás factores (Massenberg et al., 2017), mientras que los elementos del diseño de la formación, como su validez de contenido y el diseño para la transferencia, se identifican como los indicadores con mayor peso para motivar la transferencia (Grohmann et al., 2014). Es interesante también lo observado en un profuso estudio finlandés que destaca la importancia de un enfoque formativo centrado en el alumno como variable moderadora en la motivación de transferir (Gegenfurtner, 2011).

En esta misma línea, el estudio realizado por Feixas et al. (2014) aplicado a la formación de profesores considera las dimensiones tradicionales e identifica dentro de la red de factores para la transferencia una alta incidencia del diseño de formación en los cambios que evidenciaron los docentes, a la vez que la existencia de proceso de acompañamiento y el desarrollo de prácticas de innovación educativa con evidencias del trabajo realizado tienen relevancia en la vida académica de los egresados. En este estudio, además, las mejoras de su entorno de trabajo y el reconocimiento de sus jefes y compañeros mostraron alta incidencia en la transferencia (Berrocal y Campos, 2014; Cano, 2016; De Rijdt et al., 2013; Feixas et al., 2014). Para un caso de evaluación de la formación de directivos escolares se agrega la posibilidad de construir redes como un elemento importante de la transferencia de la formación (Azocar y Cano, 2015).

Finalmente, será el entorno el que permitirá observar la eficacia de un programa formativo al facilitar o 
dificultar la aplicación de las competencias desarrolladas en el contexto laboral (Salas et al., 2006). Aun cuando exista un diseño formativo orientado a la transferencia, participantes motivados por aplicar y con alta percepción de autoeficacia, se hace necesario un entorno laboral que aliente el uso de las competencias aprendidas (Grossman y Salas, 2011).

En síntesis, la posibilidad de aplicar los aprendizajes y competencias desarrollados en una acción formativa se ve afectado por la diversidad de factores arriba detallados. No depende exclusivamente de las características de los participantes, de la implementación del plan formativo ni del contexto de desempeño sino de la articulación de todos estos factores, algunos con mayor influencia que otros, pero todos intervienen en la aplicación final de los aprendizajes.

\section{Método}

El análisis se enmarcó en el curso "Liderazgo para la Innovación y el cambio en las instituciones escolares", desarrollado en modalidad b-learning para el Plan de Formación de Directores por el Ministerio de Educación chileno. Su ejecución se llevó a cabo entre los meses de noviembre 2015 y mayo de 2016, con una duración de 216 horas y contó con la participaron de docentes y directivos de la zona norte del país. El principal desafío que se propuso fue comprender, desde una experiencia de aprendizaje significativa, que el liderazgo en las instituciones educativas se ejerce entre personas e implica movilizar a la organización en torno a metas comunes, a través del desarrollo de capacidades comunicativas, juicio crítico y pensamiento creativo que oriente las acciones de innovación hacia la calidad del aprendizaje (Iranzo-García et al., 2018). El itinerario formativo se apoya en las tıc basado en un proceso de aprendizaje activo y aplicado al contexto, considerando algunas acciones claves, como el diagnóstico de necesidades y el aprendizaje de estrategias de investigación-acción. A partir de ello cada participante comienza un trabajo de toma de decisiones para su aprendizaje que les permite comprender con mayor profundidad su realidad y emprender en forma permanente estrategias para diagnosticar, mejorar y evaluar los logros de su institución.

La metodología utilizada fue de tipo secuencial mixto (Creswell y Plano, 2011), partiendo de una fase cuantitativa para identificar las principales tendencias en la transferencia de los aprendizajes por parte de los participantes del curso. Continuó con una fase cualitativa a través de un estudio de casos múltiple (Yim, 2017) que buscó comprender en mayor profundidad las variables personales y contextuales que influyeron en dicha transferencia.

Los procedimientos de levantamiento de información se llevaron a cabo 6 meses después de finalizar el curso (De Rijdt et al., 2013) a través de un cuestionario online autoadministrado. Luego, con base en los resultados se seleccionaron 12 participantes del curso en torno al cual se construyeron los casos de estudio, constituidos por el participante de la formación, su superior directo y un par profesional, a quienes se aplicaron distintas entrevistas semiestructuradas según el perfil.

\subsection{Fase cuantitativa}

Participantes. El estudio tomó como población de referencia a 68 docentes y directivos que participaron del programa formativo en Liderazgo para la Innovación y el cambio en las Instituciones Escolares. En esta fase de la investigación se trabajó con una muestra de 47 sujetos, quienes respondieron el cuestionario en línea una vez terminado el curso, logrando una muestra con un margen de error del 10\% y un 95\% de confianza. Todos los encuestados tienen formación de pedagogos y su media de experiencia en docencia es de 16 años, con una desviación estándar de 7 años. Se desempeñan en escuelas primarias y secundarias; al momento de matricularse en el curso 12 de ellos tenían un rol de directivo en el centro educativo, contando en promedio con 5 años en dicho cargo. Existe una distribución bastante homogénea entre hombres (53\%) y mujeres (47\%), quienes ejercen en establecimientos educacionales de dependencia municipal (62\%) en sectores urbanos (38\%).

Instrumentos. Se trabajó con un cuestionario autoadministrado online (Díaz y Domínguez, 2017) buscando evaluar los efectos de la formación y la aplicabilidad de los contenidos del curso en el desarrollo 
de su quehacer profesional. El instrumento consta de 23 ítems en escala Likert de 1 a 5 (Muy de acuerdo; De acuerdo; Ni de acuerdo ni en desacuerdo; En desacuerdo y Totalmente en desacuerdo), además de 10 preguntas de caracterización sociodemográfica del docente y del establecimiento donde se desempeña. Para la validación de contenido del cuestionario se recurrió al juicio de 5 expertos del ámbito del liderazgo directivo y evaluación. El cuestionario tiene una alta consistencia interna en la prueba Alfa de Cronbach (0.98). Su aplicación fue llevada a cabo luego de 6 meses de terminado el curso haciendo eco de los estudios analizados en el ámbito de la transferencia (Cano, 2016; De Rijdt et al., 2013).

Análisis. Se realizó un análisis descriptivo de todas las variables, generando 3 índices en escala positiva de 1 a 5 que dan cuenta de las principales dimensiones que se abordan en el cuestionario: autopercepción, aplicación y contexto. Por último, se realizó un análisis de correlaciones entre las principales variables y los índices construidos utilizando el estadístico Chi-cuadrado de Pearson.

\subsection{Fase cualitativa}

Informantes. Cualitativamente se trabajó con una muestra intencionada de 12 casos, compuestos por el participante de la formación, su superior directo y un compañero de trabajo par. La selección de los casos se sustentó en la información recogida en el cuestionario, construyéndose una muestra secuencial intencionada (Patton, 2015).

En la muestra se estableció como base de selección la variable estructural referida al cargo que desempeñan actualmente en el centro educativo los participantes de la formación (director, directivo, jefe de unidad técnica pedagógica o profesor de aula), su rango de edad, el nivel de aplicación de los aprendizajes (6 puntajes sobre y 6 bajo el promedio de las preguntas de aplicación observado en el cuestionario) y una distribución proporcional en género. Como se observa en la tabla I, se construyó una muestra intencionada considerando criterios que permitieran heterogeneidad y saturación en la información a recabar (Guetterman, 2015) en cuanto a características sociodemográficas y sus comportamiento respecto a la transferencia evidenciado en el cuestionario.

Tabla I. Caracterización de los casos

\begin{tabular}{lccccccc}
\hline $\begin{array}{l}\text { Cargo que } \\
\text { desempeña }\end{array}$ & Caso & Sexo & Edad & $\begin{array}{c}\text { Índice de } \\
\text { aplicación }\end{array}$ & $\begin{array}{c}\text { Puntaje } \\
\text { promedio } \\
\text { del grupo }\end{array}$ & $\begin{array}{c}\text { Diferencia } \\
\text { con media } \\
\text { grupal }\end{array}$ & $\begin{array}{c}\text { Desviación } \\
\text { típica del } \\
\text { grupo }\end{array}$ \\
\hline Director & 1 & Femenino & 52 & 5 & 4.20 & 0.80 & 1.19 \\
Escolar & 2 & Femenino & 47 & 4.78 & 4.20 & 0.58 & 1.19 \\
\hline Jefe Técnico & 3 & Masculino & 55 & 4.11 & 4.20 & -0.09 & 1.19 \\
Pedagógico & 4 & Femenino & 49 & 5 & 4.23 & 0.77 & 1.14 \\
& 6 & Masculino & 40 & 4 & 4.23 & -0.23 & 1.14 \\
\hline Inspector & 7 & Femenino & 50 & 1.33 & 4.23 & -2.90 & 1.14 \\
\hline & 8 & Masculino & 36 & 5 & 3.98 & 1.02 & 0.81 \\
Profesor & 9 & Masculino & 48 & 4.11 & 3.98 & 0.13 & 0.81 \\
de aula & 10 & Masculino & 35 & 4 & 3.98 & 0.02 & 0.81 \\
& 11 & Masculino & 47 & 3.56 & 3.98 & -0.42 & 0.81 \\
\hline
\end{tabular}

Fuente: Elaboración propia.

Cada caso fue informado por el participante de la formación, su jefe directo y un profesional par o compañero de trabajo que se desempeña dentro de la misma institución educativa. Considerando como informantes clave un total de 36 entrevistados. En cada caso tres informantes que permiten contrastar y triangular la información.

Instrumentos. Se trabajó con una entrevista semiestructurada, entendida como una variante dentro de las entrevistas cualitativas (Noboa y Robaina, 2014), partiendo de los supuestos planteados en el marco teórico, con la finalidad de profundizar en las percepciones de los entrevistados sobre la forma en que 
han sido aplicados los nuevos aprendizajes.

Análisis. Los resultados fueron trabajados por medio de análisis de contenido (Kuckartz, 2014), desarrollando una codificación selectiva de la información (Saldaña, 2013), donde se ordenaron los datos en categorías por criterios de similitud definidos previamente por el equipo de investigadores. Para luego interpretar las categorías y generar clasificaciones y agrupaciones con los principales hallazgos (Staller, 2015). Para el análisis se utilizó el software Atlas.ti.

Finalmente, se realizó una triangulación metodológica combinando los elementos del marco teórico del estudio con los resultados de los análisis cuantitativos y cualitativos para dar respuesta a los objetivos del estudio.

\section{Resultados 3.1 Fase cuantitativa}

Desde una mirada descriptiva, un primer hallazgo fue el cambio de condición de los participantes; después de seis meses de terminar la experiencia formativa el $45 \%$ de los participantes reportó un ascenso profesional dentro del mismo centro educativo o asumió algún cargo directivo en otro establecimiento educacional, los tipos de cargo asumidos son: director, inspector general, jefe de unidad técnico pedagógica o coordinador de programa.

A la vez, destacan por su nivel de aplicación las acciones en las que se involucró a otros agentes de la comunidad escolar y acciones que dieron cuenta de la motivación por generar cambios. Así, el 87.2\% señaló que ha promovido instancias de aprendizaje colaborativo y ha involucrado en los procesos de mejora a otros miembros de la comunidad educativa (89.4\%); mientras que el $85.1 \%$ de los encuestados declaró que ha identificado oportunidades de mejora en su contexto escolar y ha desarrollado algún tipo de diagnóstico para fundamentar las acciones de mejora (87.2\%).

Desde la percepción de los participantes encuestados el $85.1 \%$ está de acuerdo o muy de acuerdo con que la metodología del curso facilitó la réplica de experiencias en su desempeño laboral. Si bien los elementos que se relacionan con el diseño instruccional de la formación tienen un efecto indirecto (al afectar primeramente el aprendizaje y luego la transferencia) no implica que sea un elemento secundario (Cano, 2016), y permite comprender cómo se produce la posterior transferencia.

Como sabemos, una positiva autopercepción del aprendizaje se relaciona con la motivación para aplicar los aprendizajes, aspecto que ha sido identificado como un factor de alta relación con la transferencia (Cano, 2015; Grohmann et al., 2014; Massenberg et al., 2017). En este grupo se observa que la mayoría de los encuestados se sienten más seguros sobre su conocimiento en liderazgo directivo (95.7\%), a la vez que dicen poseer más argumentos en el ámbito del liderazgo directivo para sustentar sus propuestas (91.5\%); se sienten preparados para asumir tareas de liderazgo directivo (87.2\%), y consideran que comunican con mayor precisión sus ideas a pares y superiores (91.5\%).

En cuanto a otra variable importante para la transferencia, como es el contexto de desempeño o clima laboral (Blume et al., 2010; Salas et al., 2006), la percepción de los encuestados sobre la forma en que la comunidad educativa reconoce y valora sus acciones después de participar en el curso es positiva, pero menor que la observada en las dimensiones de autopercepción del desempeño y la aplicación de aprendizajes. El 63.8\% de los encuestados está de acuerdo o muy de acuerdo en que los directivos de su institución reconocen cambios en su desempeño asociados al curso, a la vez que el $74.5 \%$ considera que sus ideas sobre procesos de mejora son más consideradas luego de la formación recibida.

Con la finalidad de medir qué aspectos tuvieron mayor incidencia en la aplicación de aprendizajes o transferencia, a partir de las variables de cada dimensión se generaron tres índices en una escala positiva de 1 a 5. Como se aprecia en la tabla II, comparativamente los encuestados tienen una mejor percepción sobre las mejoras en su propio desempeño (autopercepción), luego sobre la aplicación de los aprendizajes en el ámbito laboral donde se desenvuelve (aplicación) y, en menor medida, sobre cómo estos cambios 
son reconocidos y valorados en su contexto laboral (contexto).

Tabla II. Estadísticos de Índices

\begin{tabular}{lccc}
\hline & Media & Mediana & SD \\
\hline Autopercepción & 4.46 & 4.8 & 0.90 \\
Aplicación & 4.14 & 4.3 & 0.96 \\
Contexto & 3.82 & 3.75 & 1.05 \\
\hline
\end{tabular}

Fuente: Elaboración propia.

Ahora bien, entendiendo que la transferencia de aprendizajes se ve influenciada fuertemente por las características del participante y el contexto de aplicación de los nuevos aprendizajes (Baldwin y Ford, 1988; Cano, 2016; Feixas et al., 2014; Grossman y Salas, 2011; Holton, 2005), se analizó la correlación existente entre los tres índices (ver tabla III) constatando una fuerte dependencia entre ellos, donde el índice de autopercepción (0.703), que incluye ítems de motivación, autoeficacia y autopercepción de aprendizaje, tiene una incidencia levemente mayor sobre la aplicación de los aprendizajes que el índice de contexto (0.688).

Tabla III. Correlación entre índices

\begin{tabular}{llccc}
\hline & & Autopercepción & Aplicación & Contexto \\
\hline Correlación & Autopercepción & 1 & 0.703 & 0.642 \\
de Pearson & Aplicación & 0.703 & 1 & 0.688 \\
& Contexto & 0.642 & 0.688 & 1 \\
\hline
\end{tabular}

Fuente: Elaboración propia.

Por su parte, el índice de contexto que incluye ítems referidos al reconocimiento institucional, acceso a recursos y cambios observados en el ambiente laboral, también tiene una correlación importante con la aplicación de los aprendizajes, como lo señala Cano (2016) a partir de su metanálisis sobre evaluación de transferencia.

Sin embargo, no se observa diferencia significativa que permita aseverar que una dimensión sea más relevante que la otra. Así, para que exista una efectiva transferencia de los aprendizajes es necesaria la articulación virtuosa entre elementos propios del participante y del contexto donde éste se desenvuelve, como lo observan Cano (2015), De Rijdt et al. (2013) y Feixas et al. (2015).

\subsection{Fase cualitativa}

Con base en el análisis de la información obtenida en las entrevistas realizadas a egresados, pares y directivos o superiores de cada caso estudiado, fue posible dar cuenta (a nivel general) de una percepción positiva del proceso de formación. Los egresados del curso manifiestan que han tenido la posibilidad no sólo de adquirir nuevos conocimientos sino también de aplicarlos en su práctica profesional, permitiendo el desarrollo de competencias o habilidades directivas. Esto es ratificado tanto por sus pares como por los superiores que fueron entrevistados.

Como resultado más significativo destaca la manifestación por parte de los egresados de un cambio de paradigma con relación a cómo deben desenvolverse en sus respectivos ambientes escolares, atribuyendo a la formación recibida su transformación en líderes o la potenciación de un liderazgo preexistente, en tanto que las dificultades detectadas se relacionan principalmente con temas estructurales de los ambientes donde desarrollan su quehacer educativo.

Luego de estas consideraciones generales, centramos el análisis aspectos relacionados con el impacto y posibilidades de transferir el aprendizaje de la formación por parte de los participantes en dos puntos: aplicación de aprendizajes y aspectos facilitadores u obstaculizadores de la transferencia. Ambos elementos se encuentran íntimamente relacionados y se han separado sólo para la articulación del relato recogido en las entrevistas. 


\subsection{Aplicación de aprendizajes}

En relación con la aplicación de conocimientos y nuevas habilidades, los participantes no sólo valoran el hecho de tener más información sino la posibilidad real de poder aplicarla en su contexto de desempeño, en consonancia con lo dicho por De Rijdt et al. (2013). Así, parte importante de la formación es que se reconoce como un aprendizaje aplicable a la realidad educativa.

Los egresados identifican la metodología, basada en un enfoque centrado en el alumno y de reflexión permanente, como un elemento que permite a los participantes hacer una lectura de su quehacer educativo cotidiano desde una visión más crítica y compleja, con exigencias de un mayor compromiso y trabajo, pero que permiten comprender con claridad la realidad escolar en la que participan e intervienen. El ejercicio del liderazgo por parte de los participantes es valorado por los aportes entregados en el curso, pero sobre todo por su aplicabilidad. Lo reconocen como aprendizajes y competencias que tienen un correlato con el ejercicio cotidiano de su quehacer, aspecto identificado en la literatura como formación en entorno real o diseño de transferencia (Burke y Hutchins, 2007; Grohmann et al., 2014; Grohmann y Kauffeld, 2013; Ornelas et al., 2016), aumentando así la de valoración positiva de los entrevistados.

Por último, el desarrollo de habilidades comunicacionales -específicamente el desarrollo de la comunicación horizontal- emerge como lo más valorado por los participantes y sus pares. Desde su punto de vista, es el elemento esencial y básico que debe estar presente en el desarrollo de habilidades directivas marcadas por un enfoque de liderazgo distribuido (Díaz et al., 2015), y que en varios casos fue posible desplegar por los participantes en sus respectivos centros educativos.

Reconociendo la dificultad de integrar más ejemplos en el artículo, cabe mencionar que en muchas expresiones de lo vertido en las entrevistas fue posible identificar cambios en la comunicación con los pares o equipos dirigidos como evidencias de un nuevo desempeño post curso, así como un nuevo énfasis en la planificación de las actividades y en la implementación de estrategias para identificar necesidades y nuevas propuestas de mejora; aspectos que posibilitaron a algunos participantes desarrollar un papel de liderazgo bajo una mirada de complejidad y capacidad crítica, abordando las problemáticas de su comunidad escolar.

\subsection{Facilitadores y obstaculizadores de la aplicación de los aprendizajes}

En la mayoría de los estudios sobre transferencia analizados (De Rijdt et al., 2013; Grossman y Salas, 2011) un elemento que toma fuerza al momento de aplicar lo aprendido al contexto profesional, es el identificado como factor institucional o de contexto, que entendemos como una condición estructural en la que se desenvuelven los participantes del curso como medio laboral. Este contexto puede favorecer o no la aplicación de los aprendizajes adquiridos en el curso y se compone de distintas dimensiones (De Rijdt et al., 2013; Gegenfurtner, 2011; Grossman y Salas, 2011), que en este estudio resumimos como la cultura organizacional de la escuela, la disposición del superior para permitir aplicaciones y la posición del participante dentro de la institución.

En relación con la cultura organizacional de la institución, resultó clave comprender en qué medida ésta sintoniza con la visión de liderazgo más constructivista que se proponía desde el programa formativo, y que luego los participantes intentaron implementar. En otros estudios este aspecto, relacionado con la validez de contenido (Grohmann y Kauffeld, 2013; Holton, 2005; Veldada et al., 2007), resulta fundamental para comprender la forma en que los egresados transfieren los nuevos aprendizajes; en este caso fue necesaria, además, la validación de los superiores jerárquicos para desplegar una nueva mirada del liderazgo.

Una segunda dimensión desde donde es posible comprender las posibilidades de transferencia de los aprendizajes, se relaciona con las características de los participantes del curso: elementos como su motivación y la percepción de autoeficacia. Es así como algunos casos, no teniendo las condiciones óptimas para implementar lo aprendido, se ajustan a los espacios y situaciones que les permiten aplicarlo, demostrando alto nivel de autoeficacia -aspecto considerado como un factor facilitador en los estudios 
sobre transferencia (Cano, 2015; De Rijdt et al., 2013; Grossman y Salas, 2011).

Por otra parte, desde la visión de los profesionales pares, fue posible observar un mejor manejo de conocimientos técnicos que se convierten en facilitadores para la aplicación de habilidades directivas aprendidas en el curso. Desde la literatura esto se considera como un elemento que ayuda a la motivación, el valor de constructo del curso que proporciona autoconfianza para aplicar (Grohmann et al., 2014; Grohmann y Kauffeld, 2013; Massenberg et al., 2017).

Algunos de los comentarios de los egresados proyectan a futuro su trabajo en la escuela teniendo como base las habilidades directivas desarrolladas, evidencia de su motivación para continuar transfiriendo lo aprendido, aspecto altamente reconocido en la literatura como factor determinante de la transferencia (Grohmann et al., 2014; Massenberg et al., 2017).

Finalmente, la posición que ocupa el egresado en el organigrama de la escuela es un elemento clave para comprender las posibilidades de implementar o no los aprendizajes y habilidades directivos desarrollados a lo largo del curso. Aquellos con cargos directivos tienen mayores posibilidades de aplicación que los que aún no lo tienen. Es Importante considerar que en algunos casos los superiores reconocen la formación recibida por los egresados y les han asignado nuevas responsabilidades al interior de la institución, posibilitando la puesta en práctica de las nuevas competencias, aspecto que evidencia la positiva valoración de los cambios observados en los egresados.

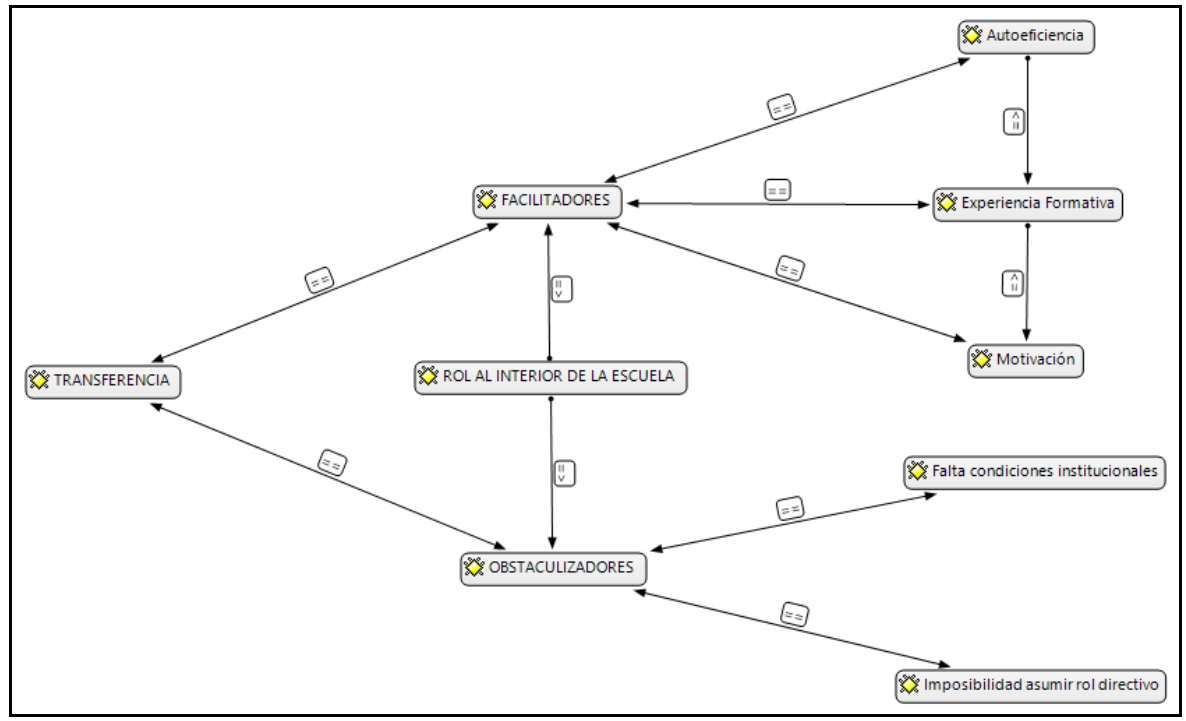

Fuente: Elaboración propia en programa AtlasTi.

Figura 1. Red semántica sobre facilitadores y obstaculizadores para transferir

Como se aprecia en la figura 1, las principales relaciones se concentran en factores que facilitan la transferencia, identificados como su percepción de autoeficacia, la experiencia formativa y su motivación para transferir, a la vez que los elementos obstaculizadores se concentran en la falta de condiciones institucionales y la imposibilidad de asumir un rol directivo en la escuela que les permita ejercitar lo aprendido en el curso.

\section{Conclusiones}

Al integrar ambos análisis, desde lo cuantitativo y lo cualitativo, es posible observar transferencia de aprendizajes sobre liderazgo directivo por parte de los participantes del curso. Desde lo declarativo, el $85 \%$ de los egresados indicó que aplica los aprendizajes del curso en su ejercicio profesional actual; desde el estudio en detalle de los 12 casos se evidencia transferencia de aprendizajes y una positiva autopercepción del desempeño posterior al curso, lo que refuerza la motivación por llevar a la práctica los 
nuevos conocimientos y se traduce en una positiva valoración por parte de pares y superiores, aspectos claves que también han sido señalados por Berrocal y Campos (2014), Blume et al. (2010), Burke y Hutchins (2007), Cano (2016), De Rijdt et al. (2013) y Salas et al. (2006).

Los aspectos relacionados con autopercepción de los egresados nos retrotraen al tema de los diseños formativos pensados en la aplicación. Prácticamente una necesidad, pero muy poco abordada, debido a que implica un cambio de paradigma en la formación y en las entidades a cargo de la formación docente. No es un requisito de las formaciones definir un diseño que se asegure de la aplicación del aprendizaje.

A partir de los resultados del cuestionario y el trabajo de campo con los 12 casos, se observa una alta valoración de contenido del plan formativo, y se considera pertinente a las exigencias de su labor en cargos directivos, haciendo transferible los nuevos aprendizajes. Es así como la validez de contenido, identificado como la utilidad percibida de lo aprendido, resulta un elemento destacable, en acuerdo con el índice de autopercepción en el análisis cuantitativo. Aspecto que desde los estudios en este ámbito (Grohmann et al., 2014; Grohmann y Kauffeld, 2013; Holton, 2005; Veldada et al., 2007) pasa a ser uno de los elementos que sustentan el alto índice de aplicación del curso.

Si bien la mayoría de los estudios analizados tratan de identificar cuál es el factor de mayor impacto en la transferencia, en este estudio se observa una interdependencia positiva entre aspectos como la autopercepción y motivación para transferir, los aprendizajes transferidos y las condiciones del contexto de aplicación. En otras palabras, existe motivación para transferir cuando el contexto lo permite, los egresados aplican los aprendizajes del curso, lo que indica que el diseño del curso, sus contenidos y metodología permiten y promueven la transferencia de los aprendizajes a su quehacer profesional (Gegenfurtner, 2011; Grohmann et al., 2014; Massenberg et al., 2017).

Mientras que las principales dificultades para transferir se relacionan con el contexto, coincidiendo con lo reportado por Salas et al. (2006) y Veldada et al., (2007). En especial en este caso, la condición del egresado de ejercer o no un cargo directivo, siendo más difícil transferir los aprendizajes para quienes no han podido ejercer funciones directivas, ya que dependen directamente de las condiciones del entorno y, especialmente, de sus superiores.

Por otro lado, las limitaciones u oportunidades dadas por el contexto educativo en el que se desempeñan los egresados juegan un importante papel en las posibilidades de aplicar los aprendizajes, y rara vez se cuenta con un real compromiso por parte de las instituciones educativas de las que provienen los participantes con la aplicación de las nuevas competencias desarrolladas.

En cuanto a las posibilidades de transferir lo aprendido a la práctica profesional, cabe señalar la importancia de que las políticas de formación incentiven y se aseguren de que el rol ejercido por el beneficiario de la formación le permitan hacer una real transferencia. Tanto en organizaciones privadas como en el caso de beneficiarios de políticas públicas de formación, como lo es este caso, el retorno de la inversión sólo será posible si el egresado ejerce un rol que le permita tomar decisiones para aplicar lo aprendido.

\section{Referencias}

Ávalos, B. (2014). La formación inicial docente en Chile: tensiones entre políticas de apoyo y control. Estudios pedagógicos, 40(Especial), 11-28. http://revistas.uach.cl/index.php/estped/article/view/2565

Ávalos, B. (2016). Learning from research on beginning teachers. En J. Loughran y M. L. Hamilton (Eds.), International Handbook of Teacher Education (pp. 487-522). Springer.

Azocar, M. y Cano, E. (2015). Evaluación diferida del plan de formación de directores, a través de la opinión de los participantes insertos en escuelas municipalizadas de la Región de los Ríos-Chile (Tesis de Máster). Universidad de Barcelona. 
Baldwin, T. T. y Ford, J. K. (1988). Transfer of training: a review and directions for future research. Personnel Psychology, 41(1), 63-105. https://doi.org/10.1111/j.1744-6570.1988.tb00632.x

Berrocal, V. y Campos, J. (2014). Resultados de la experiencia de evaluación de la transferencia de la formación: descripción del instrumento de evaluación y resultados hallados en UNED-Costa Rica. En E. Cano y A. Bartolomé (Coords.), Evaluar la formación es posible. (pp. 83-102).

http://www.Imi.ub.edu/transmedia21/pdf/7 EvaluarFormacion.pdf

Blume, B. D., Ford, J. K., Baldwin, T. T. y Huang, J. L. (2010). Transfer of training: a meta-analytic review. Journal of Management, 36(4), 1065-1105. https://doi.org/10.1177/0149206309352880

Bozu, Z. e Imbernón, F. (2016). El presente y el futuro de la formación docente permanente del profesorado universitario. Educación y Ciencia, 5(45), 94-105.

http://www.educacionyciencia.org/index.php/educacionyciencia/article/view/387

Burke, L. A. y Hutchins, H. M. (2007). Training transfer: an integrative literature review. Human Resource Development Review, 6(3), 263-296. https://doi.org/10.1177/1534484307303035

Cano, E. (2015). Evaluación de la formación. Algunas lecciones aprendidas y algunos retos de futuro. Educar, 51(1), 109-125. https://doi.org/10.5565/rev/educar.684

Cano, E. (2016). Factores Favorecedores y obstaculizadores de la transferencia de la formación del profesorado en educación superior. REICE. Revista Iberoamericana sobre Calidad, Eficacia y Cambio en Educación., 14(2), 133-150. https://doi.org/10.15366/reice2016.14.2.008

Chiaburu, D. S. y Lindsay, D. R. (2008). Can do or will do? The importance of self-efficacy and instrumentality for training transfer. Human Resource Development International, 11, 199-206.

Creswell, J. W. y Plano, V. L. (2011). Designing and conducting mixed methods research. Sage.

De Rijdt, C., Stes, A., van der Vleuten, C. y Dochy, F. (2013). Influencing variables and moderators of transfer of learning to the workplace within the area of staff development in higher education: Research review. Educational Research Review, 8, 48-74. https://doi.org/10.1016/j.edurev.2012.05.007

Díaz, C. H., Solar, M. I., Soto, V., Conejeros, M. y Vergara, J. (2015). Temas clave en la formación de profesores en Chile desde la perspectiva de docentes y directivos. Revista Complutense de Educación, 26(3), 543-569. https://doi.org/10.5209/rev RCED.2015.v26.n3.44300

Díaz, V. y Domínguez, J. A. (2017). Comparación de métodos de campo en la encuesta. Revista Española de Investigaciones Sociológicas, 158, 137-148. https://doi.org/10.5477/cis/reis.158.137

Feixas, M., Lagos, P., Fernández, I. y Sabaté, S. (2015). Modelos y tendencias en la investigación sobre efectividad, impacto y transferencia de la formación docente en educación superior. Educar, 51(1), 81108. https://www.raco.cat/index.php/Educar/article/view/287036

Feixas, M., Márquez, M. D. y Sabaté, S. (2014). Experiencia de evaluación de la transferencia de la formación del profesorado universitario: la aplicación del Cuestionario de Transferencia de la Formación Docente en la Universitat Autònoma de Barcelona. En E. Cano y A. Bartolomé (Eds.), Evaluar la formación es posible. (pp. 53-82). http://www.lmi.ub.edu/transmedia21/pdf/7 EvaluarFormacion.pdf

Gegenfurtner, A. (2011). Motivation and transfer in professional training: a meta-analysis of the moderating effects of knowledge type, instruction, and assessment conditions. Educational Research Review, 6(3), 153-168. https://doi.org/10.1016/j.edurev.2011.04.001

Grohmann, A. y Kauffeld, S. (2013). Evaluating training programs: development and correlates of the 
Questionnaire for Professional Training Evaluation. International Journal of Training and Development, 17(2), 135-155. https://doi.org/10.1111/ijtd.12005

Grohmann, A., Beller, J. y Kauffeld, S. (2014). Exploring the critical role of motivation to transfer in the training transfer process. International Journal of Training and Development, 18(2), 84-103.

https://doi.org/10.1111/ijtd.12030

Grossman, R. y Salas, E. (2011). The transfer of training: what really matters. International Journal of Training and Development, 15(2), 103-120. https://doi.org/10.1111/j.1468-2419.2011.00373.x

Guetterman, T. C. (2015). Descriptions of sampling practices within five approaches to qualitative research in education and the health sciences research in education and the health sciences. Forum: Qualitative Social Research, 16(2), 1-16. https://doi.org/10.17169/fas-16.2.2290

Holton, E. F. (2005). Holton's evaluation model: new evidence and construct elaborations. Advances in Developing Human Resources, 7(1), 37-54. https://doi.org/10.1177/1523422304272080

Hutchins, H. M., Burke, L. A. y Berthelsen, A. M. (2010). A missing link in the transfer problem? Examining how trainers learn about training transfer. Human Resource Management, 49(4), 599-618.

https://doi.org/10.1002/hrm.20371

Iranzo-García, P., Camarero-Figuerola, M., Barrios-Arós, C., Tierno-García J. y Gilabert-Medina, S. (2018). ¿Qué opinan los maestros sobre las competencias de liderazgo escolar y sobre su formación inicial ? REICE. Revista Iberoamericana sobre Calidad, Eficacia y Cambio en Educación, 16(3), 29-48.

https://doi.org/10.15366/reice2018.16.3.002

Kirkpatrick, D. L. y Kirkpatrick, J. D. (2006). Evaluating training programs: the four levels. Berrett-Koehler.

Kuckartz, U. (2014). Qualitative text analysis: a guide to methods, practice and using software. Sage.

Marcelo, C. y Vaillant, D. (2017). Políticas y programas de Inducción en la docencia en Latinoamérica. Cuadernos de Pesquisa, 47(166), 179-194. http://publicacoes.fcc.org.br/ojs/index.php/cp/article/view/4322

Massenberg, A. C., Schulte, E. M. y Kauffeld, S. (2017). Never too early: learning transfer system factors affecting motivation to transfer before and after training programs. Human Resource Development Quarterly, 28(1), 55-85. https://doi.org/10.1002/hrdq.21256

Noboa, A. y Robaina, N. (2014). Entrevista cualitativa. En A. Lucas y A. Noboa (Coods.), Conocer lo social: estrategias y técnicas de construcción y análisis de datos (347-392). Fragua.

Ornelas, D., Cordero, G. y Cano, E. (2016). La transferencia de la formación del profesorado universitario. Aportaciones de la investigación reciente. Perfiles Educativos, 38(154), 57-75.

https://doi.org/10.22201/iisue.24486167e.2016.154.57662

Patton, M. (2015). Qualitative research \& evaluation methods: Integrating theory and practice. Sage.

Salas, E., Wilson, K., Priest, H. y Guthrie, J. (2006). Training in organizations: design, delivery, and evaluation of training systems. En G. Salvendy (Ed.), Handbook of Human Factors and Ergonomics (472-512). John Wiley \& Sons.

Saldaña, J. (2013). The coding manualfor qualitative researchers. Sage.

Staller, K. M. (2015). Qualitative analysis: the art of building bridging relationships. Qualitative Social Work, 14(2), 145-153. https://doi.org/10.1177/1473325015571210 
Vaillant, D. y Marcelo, C. (2015). El ABC y D de la formación docente. Narcea.

Veldada, R., Caetano, A., Michel, J. W., Lyons, B. D. y Kavanagh, M. J. (2007). The effects of training design, individual characteristics and work environment on transfer of training. International Journal of Training and Development, 11, 282-294.

Wijk, R. V., Jansen, J. J. P. y Lyles, M. A. (2008). Inter-and intra-rganizational knowledge transfer: a meta-analytic review and assessment of its antecedents and consequences. Journal of Management Studies, 45(4), 830-853. https://doi.org/10.1111/j.1467-6486.2008.00771.x

Yim, R. K. (2017). Case study research: design and method. Sage. 